\title{
Proteomic Identification of Altered Cerebral Proteins in the Complex Regional Pain Syndrome Animal Model
}

\author{
Francis Sahngun Nahm, ${ }^{1}$ Zee-Yong Park, ${ }^{2}$ Sang-Soep Nahm, ${ }^{3}$ \\ Yong Chul Kim, ${ }^{4}$ and Pyung Bok Lee ${ }^{1}$ \\ ${ }^{1}$ Department of Anesthesiology and Pain Medicine, Seoul National University Bundang Hospital, Seongnam 463707, Republic of Korea \\ ${ }^{2}$ School of Life Science, Gwangju Institute of Science and Technology, Gwangju 500712, Republic of Korea \\ ${ }^{3}$ Laboratory of Veterinary Anatomy, College of Veterinary Medicine, Konkuk University, Seoul 143701, Republic of Korea \\ ${ }^{4}$ Department of Anesthesiology and Pain Medicine, Seoul National University Hospital, Seoul 110744, Republic of Korea
}

Correspondence should be addressed to Pyung Bok Lee; painfree@snubh.org

Received 6 March 2014; Revised 14 August 2014; Accepted 25 August 2014; Published 16 September 2014

Academic Editor: Livio Luongo

Copyright (c) 2014 Francis Sahngun Nahm et al. This is an open access article distributed under the Creative Commons Attribution License, which permits unrestricted use, distribution, and reproduction in any medium, provided the original work is properly cited.

Background. Complex regional pain syndrome (CRPS) is a rare but debilitating pain disorder. Although the exact pathophysiology of CRPS is not fully understood, central and peripheral mechanisms might be involved in the development of this disorder. To reveal the central mechanism of CRPS, we conducted a proteomic analysis of rat cerebrum using the chronic postischemia pain (CPIP) model, a novel experimental model of CRPS. Materials and Methods. After generating the CPIP animal model, we performed a proteomic analysis of the rat cerebrum using a multidimensional protein identification technology, and screened the proteins differentially expressed between the CPIP and control groups. Results. A total of 155 proteins were differentially expressed between the CPIP and control groups: 125 increased and 30 decreased; expressions of proteins related to cell signaling, synaptic plasticity, regulation of cell proliferation, and cytoskeletal formation were increased in the CPIP group. However, proenkephalin A, cereblon, and neuroserpin were decreased in CPIP group. Conclusion. Altered expression of cerebral proteins in the CPIP model indicates cerebral involvement in the pathogenesis of CRPS. Further study is required to elucidate the roles of these proteins in the development and maintenance of CRPS.

\section{Introduction}

Complex regional pain syndrome (CRPS) is a rare but serious and painful disorder. Although CRPS can occur following a minor injury, such as a sprain or even softtissue blunt trauma, severe intractable pain from CRPS can impair the quality of life. Symptoms and signs of CRPS include sensory changes (allodynia/hyperalgesia), vasomotor changes (temperature asymmetry/skin color change or asymmetry), sudomotor changes (edema/sweating change or asymmetry), and motor or trophic changes [1]. Although the exact pathophysiology of CRPS is not fully understood, several pathological mechanisms, including oxidative stress [2], neurogenic inflammation [3], and alteration in the autonomic nervous system $[4,5]$, are known to play some roles in its development. Also, psychophysical studies show that CRPS patients have distorted body image and have difficulty in recognizing the size or the position of the affected extremity [6]. The patients get worse when they think about moving the body part, even if they do not move it [7]. Mechanical stimulation of the "virtual (unaffected)" limb reflected in the mirror results in allodynia, which suggests that allodynia and paresthesia can be mediated by the brain [8]. Thus, the distorted body representation of CRPS patients can be treated with mirror therapy $[9,10]$. Also, the spreading of symptoms and signs of CRPS from the initial site of presentation to another limb is a well-known phenomenon, which may be due to aberrant central regulation of neurogenic inflammation [11]. These findings highlight the contribution of a cortical pain mechanism in patients with CRPS. Moreover, functional imaging studies provide supporting evidence for the important role of the central nervous system in the 


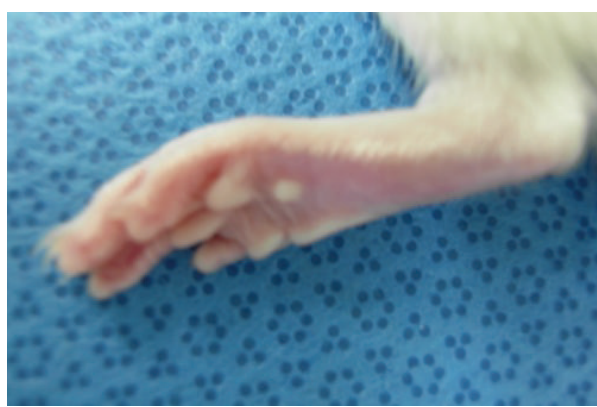

(a)

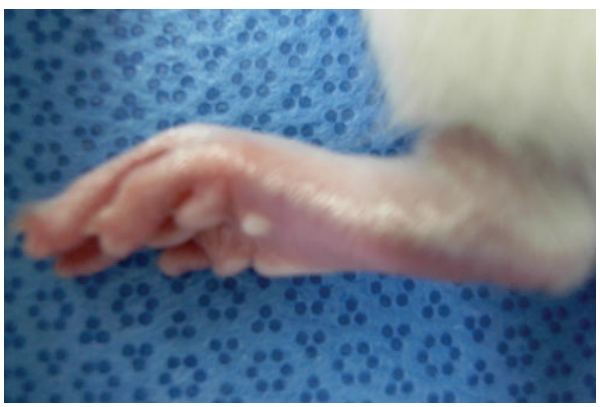

(c)

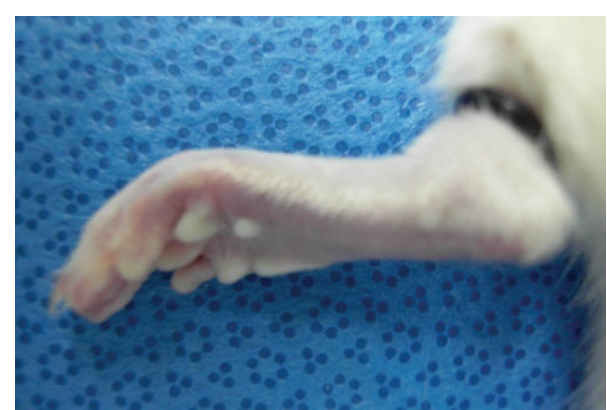

(b)

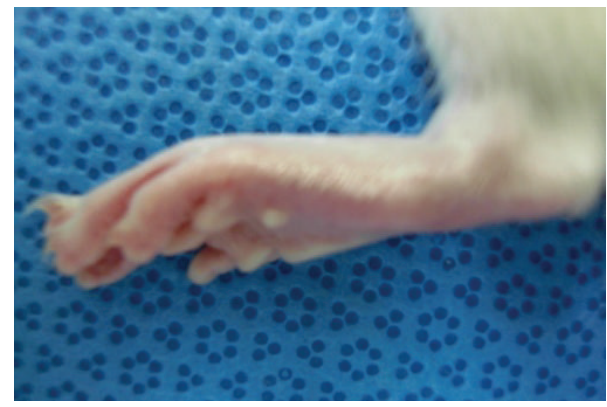

(d)

Figure 1: Plantar skin color changes in chronic postischemia pain model rats. (a) Before O-ring application, (b) during O-ring application, (c) 1 hour after reperfusion, and (d) $4 \mathrm{~h}$ after reperfusion.

pathogenesis of CRPS [12-14], and recent research suggests that changes in cortical structures can contribute to the pathophysiology of CRPS [15].

Thus, the brain seems to play an important role in the development and maintenance of symptoms and signs in patients with CRPS. Some researchers insist that the peripheral changes in CRPS must be understood as a manifestation of changes in the brain [16]. Therefore, we postulated that protein expression would be altered in the CRPS-affected brain. However, there have been no studies on the changes of cerebral protein expression in CRPS. Therefore, to verify our hypothesis, we conducted a proteomic analysis using multidimensional protein identification technology (MudPIT) in a chronic postischemia perfusion (CPIP) rat model, a novel and widely used experimental model of CRPS type 1 [17].

\section{Materials and Methods}

2.1. Animals. This study was approved by the Institutional Animal Care and Use Committee of Seoul National University Bundang Hospital (IACUC number 52-2009-033). Male Sprague-Dawley rats weighing 200-250 g had free access to food and water and were housed individually in cages with soft bedding under a $12 \mathrm{~h}$ night/day light cycle at a constant temperature of $20-22^{\circ} \mathrm{C}$ and a humidity level of $55-60 \%$. The animals were acclimatized for at least 1 week prior to the CPIP procedure.

2.2. CPIP Model Generation. The CPIP animal model was generated according to previous methods [17]. Briefly, after induction of anesthesia with isoflurane, a tight fitting Oring (O-ring West, Seattle, WA, USA) with a $5.5 \mathrm{~mm}$ internal diameter was applied to the left hind limb of each anesthetized rat just proximal to the left ankle joint for $3 \mathrm{~h}$. The O-ring was then removed from the anesthetized rat, allowing reperfusion of the hind limb (Figure 1). The animals in the control group underwent anesthesia similar to the CPIP animals, but the O-ring was not placed around the hind limb.

2.3. Behavioral Tests. All behavioral tests were performed during the daylight portion of the regulated circadian cycle between 9 a.m. and 3 p.m. To assess the mechanical threshold, the rats were placed in individual plastic cages with wire mesh bottoms. After 20 min acclimatization, calibrated von Frey filaments (Stoelting Co., Wood Dale, IL, USA) with logarithmically increasing stiffness of $0.41,0.70,1.20,2.00$, $3.63,5.50,8.50$, and $15.10 \mathrm{~g}$ were applied to the midplantar surface of the hind paw. The mechanical threshold was assessed using an up-down statistical method [18]. Then, the change in the mechanical threshold (CMT, \%) was calculated. The mechanical threshold was examined during the postreperfusion period: $1 \mathrm{~h}, 4 \mathrm{~h}, 24 \mathrm{~h}, 48 \mathrm{~h}$, day 7 , and day 21. The CMT was calculated by following equation:

$$
\operatorname{CMT}(\%)=\frac{M_{\text {post }}-M_{\text {pre }}}{M_{\text {pre }}} \times 100 .
$$

We used the findings from the neurobehavioral test on day 21 to classify the animals into groups: rats whose CMT was decreased $50 \%$ or more after the CPIP procedure were classified as the successful CPIP (A) group. The mechanical 
threshold of the animals in the control (C) group was also examined and compared using repeated-measures analysis of variance. All animals were sacrificed 3 weeks after the CPIP procedure for proteomic analysis.

2.4. Proteomic Analysis. The difference in cerebral protein expression between Groups A and $\mathrm{C}$ was explored using a MudPIT as follows.

2.4.1. Protein Extraction. A total of six animals (three from Groups A and C) were used for the mass spectrometry analysis. On the day 21 , right half of each rat cerebrum was grinded using a mortar in liquid nitrogen. The tissue powder was kept at $-80^{\circ} \mathrm{C}$. The tissue powder was resolubilized in a small volume of $8 \mathrm{M}$ urea, $100 \mathrm{mM}$ Tris- $\mathrm{HCl}, \mathrm{pH} 8.5$, and $1 \mathrm{mM}$ dithiothreitol (DTT) for two hours. The homogenates were sonicated and centrifuged at $100,000 \times \mathrm{g}$ for $1 \mathrm{~h}$. Next, $5 \mathrm{mM}$ DTT was added to the homogenate for $30 \mathrm{~min}$ at $37^{\circ} \mathrm{C}$ and alkylated with $25 \mathrm{mM}$ iodoacetamide for $30 \mathrm{~min}$ at $37^{\circ} \mathrm{C}$ in the dark. The samples were then diluted with $2 \mathrm{M}$ urea and with $50 \mathrm{mM}$ Tris- $\mathrm{HCl}, \mathrm{pH} 8.0$, and digested at $37^{\circ} \mathrm{C}$ overnight with sequence grade trypsin (Promega Co., Fitchburg, MA, USA) diluted 1:50 in $5 \mathrm{mM} \mathrm{CaCl}_{2}$.

2.4.2. MudPIT. Peptides were separated with an Agilent 1100 series high-performance liquid chromatography (HPLC) pump (Agilent technologies, Santa Clara, CA, USA) connected to a linear quadruple ion-trap mass spectrometer (MS, LTQ, Thermo-Finnigan, San Jose, CA, USA) using an inhouse-built nanoelectrospray ionization interface. To identify peptides, the ion-trap mass spectrometer was operated in a data-dependent MS/MS mode $(m / z 400-2000)$, in which a full MS scan was followed by $10 \mathrm{MS} / \mathrm{MS}$ scans and the temperature of the heated capillary was $200^{\circ} \mathrm{C}$. MS/MS spectra were generated in the positive ion mode at an electrospray voltage of $2.5 \mathrm{kV}$ and normalized collision energy of $35 \%$. An analytical column-fused (100 $\mu$ m internal diameter) silica capillary microcolumn (Polymicro technologies, Phoenix, AZ, USA) was pulled to a fine tip using a laser puller and packed with $7 \mathrm{~cm}$ of $5 \mu \mathrm{m} \mathrm{C18}$ reverse-phase resin, which was connected to an internal diameter of $250 \mu \mathrm{m}$ fused-silica trapping column packed with $2 \mathrm{~cm}$ of SCX followed by $2 \mathrm{~cm}$ of C18 resin. Each $30 \mu \mathrm{g}$ peptide mixture was manually loaded onto separate columns using a pressure vessel. A sevenstep chromatography run was carried out on each sample and three buffers were used (buffer A: $5 \% \mathrm{ACN} / 0.1 \%$ formic acid, buffer B: $80 \% \mathrm{ACN} / 0.1 \%$ formic acid, and buffer C: $5 \%$ ACN/0.1\% formic acid/500 mM ammonium acetate).

2.4.3. Data Searching and Analysis. Acquired MS/MS spectra were searched against an international protein index "rat v. 3.78 FASTA-format decoy database" downloaded from European Bioinformatics Institute (EBI, http://www.ebi.ac.uk/). The SEQUEST algorithm [19] was used to find the best matching sequences from the database with BioWorks 3.3 (Thermo Fisher Scientific Inc., Rockford, IL, USA) for fully tryptic peptides. The mass of the amino acid cysteine was statically modified by $+57 \mathrm{Da}$ and the differential modification search

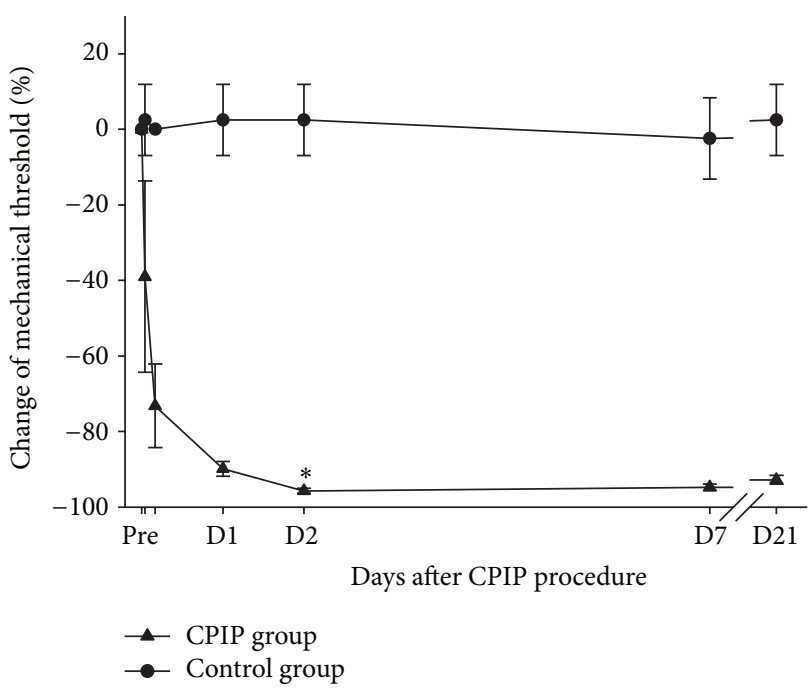

FIGURE 2: Change in mechanical threshold (\%). Group A showed a significant decrease in mechanical threshold compared to group $\mathrm{C}$ $\left(n=7\right.$ in each group). Asterisk $\left(^{*}\right)$ indicates $P<0.05$ at each time point. Group A: CPIP group and Group C: control group.

was performed for oxidation (+16 Da on Met). Xcorr values were based on tryptic peptides and charge states following 1.8 for singly charged peptides, 2.5 for doubly charged peptides, 3.5 for triply charged peptides, and 0.08 for $\Delta \mathrm{Cn}$ (DTASelect v. 2.0.39). The analysis of protein fold-change was quantified by an overall spectral counting method comparison of labelfree methods for quantifying human proteins [20].

\section{Results}

3.1. Behavioral Tests. A total of 14 animals $(n=7$ per group) were included in the behavioral test. Before the CPIP procedure, there were no differences in the mechanical threshold between the groups. However, Group A exhibited a significant decrease in the mechanical threshold compared to Group C after the CPIP procedure $(P<0.01$, Figure 2$)$. The mean differences of CMT (\%) in Group A compared to Group C were $-41.5,-73.2,-92.3,-98.2,-92.2$, and -95.3 after CPIP procedure $1 \mathrm{~h}, 4 \mathrm{~h}$, day 1 , day 2 , day 7 , and day 21 , respectively.

3.2. Differential Protein Expression in the Rat Cerebrum. A total of 454 proteins were differentially expressed between Groups A and C under the criterion of $P$ value $<0.1$. Among the 454 proteins, we selected those found in the cerebrum of all study animals in either group and excluded "uncharacterized proteins" and "hypothetical proteins." Finally, we found 155 differentially expressed proteins between Group A and Group C: 125 increased (Table 6) and 30 decreased (Table 7). Specifically, expression of proteins related to cell signaling (Table 1), synaptic plasticity (Table 2), regulation of cell proliferation (Table 3), and cytoskeletal formation (Table 4) was increased in Group A. Also, expression of a group of protein kinases (calmodulin dependent protein kinase II beta $\mathrm{M}$ isoform, casein kinase 2, phosphoenolpyruvate carboxykinase 2 , mitogen-activated protein kinase 4 , protein 
TABLE 1: Increased cerebral proteins in the chronic post-ischemia pain group; proteins which might be related to cell signaling.

\begin{tabular}{|c|c|c|c|}
\hline Number & Symbol & Description & $P$ value \\
\hline 1 & Kctd12 & Potassium channel tetramerisation domain containing 12 & 0.004 \\
\hline 2 & Ecsit & Evolutionarily conserved signaling intermediate in Toll pathway, mitochondrial & 0.004 \\
\hline 3 & Tns1 & Tensin 1 & 0.004 \\
\hline 4 & Ccbp2 & Chemokine-binding protein 2 & 0.004 \\
\hline 5 & Apbal & Amyloid beta A4 precursor protein-binding family A member 1 & 0.008 \\
\hline 6 & Tnc & Tenascin C & 0.008 \\
\hline 7 & Rabl2b & $\mathrm{RAB}$, member of RAS oncogene family-like $2 \mathrm{~B}$ & 0.008 \\
\hline 8 & Epha4 & Eph receptor A4 & 0.035 \\
\hline 9 & Rab6a & Ras-related protein Rab-6A & 0.038 \\
\hline 10 & Gpr158 & G protein-coupled receptor 158 & 0.042 \\
\hline 11 & Anxa2 & Isoform Short of Annexin A2 & 0.043 \\
\hline 12 & Hgs & Isoform 1 of hepatocyte growth factor-regulated tyrosine kinase substrate & 0.054 \\
\hline 13 & Prkcd & Isoform 1 of protein kinase $\mathrm{C}$ delta type & 0.060 \\
\hline 14 & Gabarapl2 & Gamma-aminobutyric acid receptor-associated protein-like 2 & 0.065 \\
\hline 15 & Map2k4 & Dual specificity mitogen-activated protein kinase 4 & 0.066 \\
\hline 16 & Cacng2 & Voltage-dependent calcium channel gamma-2 subunit & 0.083 \\
\hline 17 & $\mathrm{Phb} 2$ & Prohibitin-2 & 0.086 \\
\hline 18 & Camk2b & Calmodulin-dependent protein kinase II beta $\mathrm{M}$ isoform & 0.086 \\
\hline 19 & Anxa5 & Annexin A5 & 0.093 \\
\hline 20 & Scn2al & Sodium channel Nav1.2 & 0.095 \\
\hline 21 & Rab10 & Ras-related protein Rab-10 & 0.097 \\
\hline
\end{tabular}

TABLE 2: Increased cerebral proteins in the chronic postischemia pain group; proteins which might be related to synaptic plasticity.

\begin{tabular}{lclr}
\hline Number & Symbol & Description & $P$ value \\
\hline 1 & Itpr2 & Inositol 1,4,5-trisphosphate receptor type 2 & 0.001 \\
2 & Kctd12 & Potassium channel tetramerisation domain containing 12 & 0.004 \\
3 & Grid2 & Glutamate receptor delta-2 subunit & 0.004 \\
4 & Baiap3 & BAIl-associated protein 3-like isoform 2 & 0.008 \\
5 & Atad1 & ATPase family, AAA domain containing 1 & 0.008 \\
6 & Pick1 & PRKCA-binding protein & 0.008 \\
7 & Nlgn3 & Isoform 1 of Neuroligin-3 & 0.056 \\
8 & Nudc & Nuclear migration protein nudC & 0.085 \\
9 & Camk2b & Calmodulin-dependent protein kinase II beta M isoform & 0.086 \\
\hline
\end{tabular}

TABLE 3: Increased cerebral proteins in the chronic postischemia pain group; proteins which might be related to regulation of cell proliferation.

\begin{tabular}{lcll}
\hline Number & Symbol & Description & $P$ value \\
\hline 1 & Pik3r4 & Phosphoinositide 3-kinase regulatory subunit 4 & 0.001 \\
2 & Itpr2 & Inositol 1,4,5-trisphosphate receptor type 2 & 0.001 \\
3 & Anp32b & Acidic leucine-rich nuclear phosphoprotein 32 family member B & 0.002 \\
4 & Plk1 & Serine/threonine-protein kinase & 0.004 \\
5 & Drg2 & Developmentally regulated GTP binding protein 2-like & 0.004 \\
6 & Dmwd & Dystrophia myotonica-containing WD repeat motif & 0.008 \\
7 & Acin1 & Apoptotic chromatin condensation inducer 1 protein & 0.008 \\
8 & Pole2 & Polymerase (DNA directed), epsilon 2 & 0.008 \\
9 & Cyld & Ubiquitin carboxyl-terminal hydrolase & 0.076 \\
10 & Csnk2a2 & Casein kinase 2, alpha prime polypeptide & 0.084 \\
11 & Rab10 & Ras-related protein Rab-10 & 0.097 \\
\hline
\end{tabular}


TABLE 4: Increased cerebral proteins in the chronic postischemia pain group; proteins which might be related to cytoskeletal formation.

\begin{tabular}{lclc}
\hline Number & Symbol & Description & $P$ value \\
\hline 1 & Krt4 & Keratin, type II cytoskeletal 4 & 0.008 \\
2 & Sntb2 & Syntrophin, beta 2 & 0.008 \\
3 & Ckap5 & Cytoskeleton associated protein 5 & 0.038 \\
4 & Fermt2 & Fermitin family homolog 2 & 0.063 \\
5 & Cotl1 & Coactosin-like protein & 0.065 \\
6 & Rps5 & 40S ribosomal protein S5 & 0.072 \\
7 & Colla2 & Collagen alpha-2(I) chain & 0.079 \\
8 & Actr10 & Actin-related protein 10 homolog & 0.080 \\
9 & Etl4 & Enhancer trap locus 4-like & 0.083 \\
10 & Farp1 & FERM, RhoGEF (Arhgef), and pleckstrin domain protein 1 & 0.094 \\
\hline
\end{tabular}

TABLE 5: Decreased cerebral proteins in the chronic postischemia pain group; proteins which might be related to cell signaling.

\begin{tabular}{lclr}
\hline Number & Symbol & Description & $P$ value \\
\hline 1 & Vwa1 & Von Willebrand factor A domain-containing protein 1 & 0.002 \\
2 & Ppplr10 & Serine/threonine-protein phosphatase 1 regulatory subunit 10 & 0.003 \\
3 & Slc4a8 & Isoform 2 of electroneutral sodium bicarbonate exchanger 1 & 0.003 \\
4 & Daam2 & Dishevelled associated activator of morphogenesis 2 \\
5 & Trim32 & Tripartite motif protein 32 & 0.003 \\
6 & Slcla1 & Excitatory amino acid transporter 3 \\
7 & Spn & Sialophorin & 0.007 \\
8 & Crbn & Cereblon & 0.007 \\
9 & Thoc1 & Da2-19 THO complex subunit 1 \\
10 & Lmo7 & Lim domain only protein 7 \\
11 & Rps27a & Ribosomal protein S27a \\
12 & Sema4d & Sema domain & 0.007 \\
13 & Sec3l1 & SEC3-like 1 & 0.007 \\
14 & Spna2 & Alpha II spectrin & 0.007 \\
15 & Pde10a & Isoform 3 of cAMP- and cAMP-inhibited cGMP 3', $5^{\prime}$-cyclic phosphodiesterase 10A \\
16 & Snx2 & Sorting nexin 2 & 0.007 \\
17 & Slc25a3 & Phosphate carrier protein, mitochondrial \\
18 & Cox6a1 & Cytochrome c oxidase subunit 6A1, mitochondrial & 0.007 \\
\hline
\end{tabular}

kinase C delta, N-terminal kinase like protein, uridine kinaselike 1, serine/threonine protein kinase PLK 1, and phosphoinositide 3 kinase regulatory subunit 4) and calcium-related proteins (inositol 1,4,5-triphosphate receptor type 2, annexin $\mathrm{A} 1$, annexin $\mathrm{A} 2$, annexin $\mathrm{A} 5$, voltage-dependent $\mathrm{Ca}^{2+}$ channel gamma- 2 subunit, and voltage-dependent $\mathrm{Ca}^{2+}$ channel beta3 subunit, and coiled-coil domain-containing protein 47) was also elevated in Group A. However, several proteins were decreased in group A. Specifically, expression of proteins related to cell signaling (Table 5) and metabolism of fatty acid (peroxisomal 3,2-trans-enoyl Co A isomerase, acetylCoA acyltransferase $1 \mathrm{~b}$, and acetyl-CoA acetyltransferase 2) were decreased. Also, proenkephalin A, protein cereblon, and neuroserpin were decreased in Group A.

\section{Discussion}

In our study, various proteins were differentially expressed in the cerebrum of CPIP animals. Specifically, expressions of proteins related to cell signaling, synaptic plasticity, regulation of cell proliferation, and cytoskeletal formation were increased in Group A. These findings suggest that both functional and structural changes may occur in the cerebrum of CPIP animals, and altered protein expression can be related to the development of CRPS. This is the first study of cerebral protein expression changes in the CPIP rat model.

We also found that inositol 1,4,5-triphosphate (IP3) receptor type 2 and phosphoinositide 3 kinase (PI3K) regulatory subunit were also increased in Group A. IP3 receptor is intracellular calcium release channel and is regulated by calcium and calmodulin (CaM) [21]. And it is known that $\mathrm{PI} 3 \mathrm{~K}$ is an important mediator of central sensitization in painful inflammatory condition [22], and many tumorous conditions are related to this enzyme $[23,24]$. Based on these findings, cerebral overexpression of IP3 receptor type 2 and $\mathrm{PI} 3 \mathrm{~K}$ can be related to the sustained pain after rat CPIP model. 
TABLE 6: Cerebral proteins with increased expression in the chronic postischemia pain (CPIP) group.

\begin{tabular}{|c|c|c|c|}
\hline Number & Symbol & Description & $P$ value \\
\hline 1 & Itpr2 & Inositol 1,4,5-trisphosphate receptor type 2 & 0.001 \\
\hline 2 & Pik3r4 & Phosphoinositide 3-kinase regulatory subunit 4 & 0.001 \\
\hline 3 & Exoc7 & Exocyst complex component 7 & 0.001 \\
\hline 4 & Rcor2 & REST corepressor 2 & 0.001 \\
\hline 5 & Anp32b & Acidic leucine-rich nuclear phosphoprotein 32 family member B & 0.002 \\
\hline 6 & Qrich2 & Glutamine rich 2-like & 0.002 \\
\hline 7 & Dnah11 & Dynein, axonemal, heavy chain 11 & 0.002 \\
\hline 8 & Plk1 & Serine/threonine-protein kinase PLK1 & 0.004 \\
\hline 9 & Ephxl & Epoxide hydrolase 1 & 0.004 \\
\hline 10 & Cacnb3 & Voltage-dependent L-type calcium channel subunit beta-3 & 0.004 \\
\hline 11 & Anxal & Annexin Al & 0.004 \\
\hline 12 & Tns1 & Tensin 1 & 0.004 \\
\hline 13 & Hdac4 & Histone deacetylase 4 & 0.004 \\
\hline 14 & Osbpl7 & Oxysterol binding protein like 7 & 0.004 \\
\hline 15 & Ecsit & Evolutionarily conserved signaling intermediate in Toll pathway, mitochondrial & 0.004 \\
\hline 16 & Sorbs3 & Sorbin and SH3 domain containing 3, isoform CRA_b & 0.004 \\
\hline 17 & Kctd12 & Potassium channel tetramerisation domain containing 12 & 0.004 \\
\hline 18 & Ccbp2 & Chemokine-binding protein 2 & 0.004 \\
\hline 19 & Drg2 & Developmentally regulated GTP binding protein 2-like & 0.004 \\
\hline 20 & Grid2 & Glutamate receptor delta-2 subunit & 0.004 \\
\hline 21 & Safb & Scaffold attachment factor B1 & 0.008 \\
\hline 22 & Dnm3 & Isoform 1 of Dynamin-3 & 0.008 \\
\hline 23 & Dnajc16 & DnaJ homolog subfamily $\mathrm{C}$ member 16 & 0.008 \\
\hline 24 & Sntb2 & Syntrophin, beta 2 & 0.008 \\
\hline 25 & Pnpt1 & Polyribonucleotide nucleotidyltransferase 1 & 0.008 \\
\hline 26 & Eif3g & Eukaryotic translation initiation factor 3 subunit $G$ & 0.008 \\
\hline 27 & Pole2 & Polymerase (DNA directed), epsilon 2 & 0.008 \\
\hline 28 & Scyll & N-terminal kinase-like protein & 0.008 \\
\hline 29 & Atad1 & ATPase family, AAA domain containing & 0.008 \\
\hline 30 & Krt4 & Keratin, type II cytoskeletal 4 & 0.008 \\
\hline 31 & Ctsa & Protective protein for beta-galactosidase & 0.008 \\
\hline 32 & Abcal & 5 ATP-binding cassette, subfamily A (ABC1), member 15 & 0.008 \\
\hline 33 & Dmwd & Dystrophia myotonica-containing WD repeat motif & 0.008 \\
\hline 34 & Baiap3 & BAIl-associated protein 3 -like isoform 2 & 0.008 \\
\hline 35 & Znf512b & Uridine kinase-like 1 & 0.008 \\
\hline 36 & Gale & Gale protein & 0.008 \\
\hline 37 & Pick1 & PRKCA-binding protein & 0.008 \\
\hline 38 & Acin1 & Acinl protein & 0.008 \\
\hline 39 & Chid1 & Chitinase domain containing 1 & 0.008 \\
\hline 40 & Pcyoxll & Pcyoxll protein & 0.008 \\
\hline 41 & Rabl2b & RAB, member of RAS oncogene family-like $2 \mathrm{~B}$ & 0.008 \\
\hline 42 & Serpina3k & Serine protease inhibitor $\mathrm{A} 3 \mathrm{~K}$ & 0.008 \\
\hline 43 & Glg1 & Golgi apparatus protein 1 & 0.008 \\
\hline 44 & Tnc & Tenascin C & 0.008 \\
\hline 45 & Lysmd1 & LysM and putative peptidoglycan-binding domain-containing protein 1 & 0.008 \\
\hline 46 & Apbal & Amyloid beta A4 precursor protein-binding family A member 1 & 0.008 \\
\hline 47 & Ckap5 & Cytoskeleton associated protein 5 & 0.038 \\
\hline 48 & Ndufab1 & Acyl carrier protein & 0.035 \\
\hline 49 & Epha4 & Eph receptor A4 & 0.035 \\
\hline 50 & Kalrn & Isoform 2 of Kalirin & 0.035 \\
\hline 51 & Myh14 & Myosin, heavy chain 14 & 0.035 \\
\hline
\end{tabular}


TABle 6: Continued.

\begin{tabular}{|c|c|c|c|}
\hline Number & Symbol & Description & $P$ value \\
\hline 52 & Anxa2 & Isoform Short of Annexin A2 & 0.043 \\
\hline 53 & Ccdc47 & Coiled-coil domain-containing protein 47 & 0.043 \\
\hline 54 & Gpr158 & G protein-coupled receptor 158 & 0.042 \\
\hline 55 & Cugbp1 & CUGBP Elav-like family member 1 & 0.041 \\
\hline 56 & $\mathrm{Hba} 2$ & Hemoglobin alpha 2 chain & 0.040 \\
\hline 57 & Acsl3 & Isoform long of long-chain-fatty-acid-CoA ligase 3 & 0.040 \\
\hline 58 & Rab6a & Ras-related protein Rab-6A & 0.038 \\
\hline 59 & $\mathrm{Hbb}$ & Hemoglobin subunit beta-1 & 0.048 \\
\hline 60 & Hbb-b1 & Zero beta-1 globin & 0.044 \\
\hline 61 & Khsrp & Far upstream element-binding protein 2 & 0.043 \\
\hline 62 & Scamp5 & Secretory carrier-associated membrane protein 5 & 0.048 \\
\hline 63 & Aldh3a2 & Fatty aldehyde dehydrogenase & 0.049 \\
\hline 64 & Mesdc2 & LDLR chaperone MESD & 0.049 \\
\hline 65 & Rab3d & GTP-binding protein Rab-3D & 0.051 \\
\hline 66 & Vps29 & Isoform 2 of vacuolar protein sorting-associated protein 29 & 0.051 \\
\hline 67 & Psma31 & Psma3 Proteasome subunit alpha type- 3 & 0.053 \\
\hline 68 & Hgs & Isoform 1 of hepatocyte growth factor-regulated tyrosine kinase & 0.054 \\
\hline 69 & Nlgn3 & Isoform 1 of neuroligin-3 & 0.056 \\
\hline 70 & Cygb & Cytoglobin & 0.060 \\
\hline 71 & Pcsk2 & Neuroendocrine convertase 2 & 0.060 \\
\hline 72 & Prkcd & Isoform 1 of Protein kinase $\mathrm{C}$ delta & 0.060 \\
\hline 73 & Fnbp4 & Formin binding protein 4 & 0.062 \\
\hline 74 & Eif2s $3 x$ & Eukaryotic translation initiation factor 2 subunit 3 & 0.063 \\
\hline 75 & Fermt2 & Fermitin family homolog 2 & 0.063 \\
\hline 76 & Vps33a & Vacuolar protein sorting-associated protein 33A & 0.063 \\
\hline 77 & SNX3 & Sorting nexin-3 & 0.063 \\
\hline 78 & Exoc8 & Exocyst complex component 8 & 0.063 \\
\hline 79 & Thrap3 & Thyroid hormone receptor-associated protein 3 & 0.063 \\
\hline 80 & Ndufa1 & NADH dehydrogenase (ubiquinone) 1 alpha subcomplex, 1 & 0.063 \\
\hline 81 & Gabarapl2 & Gamma-aminobutyric acid receptor-associated protein-like 2 & 0.065 \\
\hline 82 & Cotl1 & Coactosin-like protein & 0.065 \\
\hline 83 & Gad1 & Glutamate decarboxylase 1 & 0.065 \\
\hline 84 & Ehd1 & EH domain-containing protein 1 & 0.066 \\
\hline 85 & Map2k4 & Mitogen-activated protein kinase 4 & 0.066 \\
\hline 86 & Mug1 & Murinoglobulin (alpha-1-inhibitor 3) & 0.070 \\
\hline 87 & Pck2 & Phosphoenolpyruvate carboxykinase 2 & 0.072 \\
\hline 88 & Rps5 & 40 S ribosomal protein S5 & 0.072 \\
\hline 89 & Ap2s1 & Adaptor protein complex 2 subunit sigma & 0.075 \\
\hline 90 & Tpp1 & Tripeptidyl-peptidase 1 & 0.076 \\
\hline 91 & Cyld & Ubiquitin carboxyl-terminal hydrolase & 0.076 \\
\hline 92 & Nuc & Nucleolin-like protein & 0.079 \\
\hline 93 & Colla2 & Collagen alpha-2(I) chain & 0.079 \\
\hline 94 & Slc6a17 & Orphan sodium- and chloride-dependent neurotransmitter transporter NTT4 & 0.079 \\
\hline 95 & Actr10 & Actin-related protein 10 homolog & 0.080 \\
\hline 96 & Cacng2 & Voltage-dependent calcium channel gamma-2 subunit & 0.083 \\
\hline 97 & Ampd3 & AMP deaminase 3 & 0.083 \\
\hline 98 & Eif5b-ps1 & Eif5b Eukaryotic translation initiation factor 5B & 0.083 \\
\hline 99 & Timm9 & Mitochondrial import inner membrane translocase subunit Tim9 & 0.083 \\
\hline 100 & Etl4 & Enhancer trap locus 4-like & 0.083 \\
\hline 101 & Csnk2a2 & Casein kinase 2, alpha prime polypeptide & 0.084 \\
\hline 102 & Cct6a & Chaperonin containing TCP1 subunit $6 \mathrm{a}$ & 0.084 \\
\hline 103 & Nudc & Nuclear migration protein nud & 0.085 \\
\hline
\end{tabular}


TABLE 6: Continued.

\begin{tabular}{|c|c|c|c|}
\hline Number & Symbol & Description & $P$ value \\
\hline 104 & Ndufa13 & NADH dehydrogenase (ubiquinone) 1 alpha subcomplex 13 & 0.085 \\
\hline 105 & Camk2b & Calmodulin-dependent protein kinase II beta $\mathrm{M}$ isoform & 0.086 \\
\hline 106 & Clta & Isoform brain of clathrin light chain A & 0.086 \\
\hline 107 & Asahl & Acid ceramidase & 0.086 \\
\hline 108 & Phb2 & Prohibitin-2 & 0.086 \\
\hline 109 & Sod1 & Superoxide dismutase $[\mathrm{Cu}-\mathrm{Zn}]$ & 0.088 \\
\hline 110 & Ndufs8 & NADH dehydrogenase (ubiquinone) 1 alpha subcomplex subunit 8 & 0.090 \\
\hline 111 & Slc17a7 & Isoform 1 of vesicular glutamate transporter 1 & 0.091 \\
\hline 112 & Ugp2 & UDP-glucose pyrophosphorylase 2, isoform CRA-b & 0.091 \\
\hline 113 & Rala & Ras-related protein Ral-A & 0.091 \\
\hline 114 & Anxa5 & Annexin A5 & 0.093 \\
\hline 115 & Hnrph1 & Isoform 1 of heterogeneous nuclear ribonucleoprotein $\mathrm{H}$ & 0.093 \\
\hline 116 & Stxbp5l & Syntaxin binding protein 5-like & 0.093 \\
\hline 117 & $\mathrm{Abcd} 3$ & ATP-binding cassette subfamily D member 3 & 0.094 \\
\hline 118 & Farp1 & FERM, RhoGEF (Arhgef), and pleckstrin domain protein 1 & 0.094 \\
\hline 119 & Leng4 & Leng4 protein & 0.094 \\
\hline 120 & Scn2al & Sodium channel Nav1.2 & 0.095 \\
\hline 121 & Rab10 & Ras-related protein Rab-10 & 0.097 \\
\hline 122 & Aldh7a1 & Alpha-aminoadipic semialdehyde dehydrogenase & 0.097 \\
\hline 123 & Cltb & Isoform Brain of Clathrin light chain B & 0.097 \\
\hline 124 & Phyhipl & Isoform 1 of phytanoyl-CoA hydroxylase-interacting protein-like & 0.098 \\
\hline 125 & Synpo & Isoform 1 of synaptopodin & 0.099 \\
\hline
\end{tabular}

Among the 155 proteins expressed differentially, calcium related proteins, including calcium calmodulin kinase II (CaMKII), were increased in group A. Calcium plays a crucial role in many physiological processes, including signal transduction, cell growth, and proliferation. CaMKII is one of the most prominent protein kinases, present in every tissue, but most concentrated in the brain. CaMKII plays various roles, including synthesis and release of neurotransmitter modulation of ion channel activity, synaptic plasticity, learning, and memory [25-28]. Moreover, CaMKII is thought to be important in central sensitization [29-31] and is implicated in central neuropathic pain [31] and long term potentiation (LTP) [32]. LTP is initiated when NMDA receptors allow $\mathrm{Ca}^{2+}$ into the postsynaptic neuron, and this $\mathrm{Ca}^{2+}$ influx activates CaMKII. LTP in nociceptive spinal pathways shares several features with hyperalgesia, and LTP at synapses constitutes a contemporary cellular model for pain $[33,34]$. And it was reported that the overexpression of CaMKII was observed in the dorsal root ganglia of rat model of type 1 diabetes [35], and the inhibition of CaMKII can reverse the chronic inflammatory pain [36]. These findings are consistent with the result of our study. Therefore, overexpression of cerebral CaMKII implicates cerebral involvement in CRPS, and CaMKII can be a target for the prevention and treatment of CRPS.

In addition, we observed that proenkephalin A, cereblon, and neuroserpin decreased in CPIP animals. Proenkephalin is an endogenous opioid hormone which produces the enkephalin peptide. Enkephalin provides a role as inhibiting neurotransmitters in the pathway for pain perception to reduce pain perception. Therefore, decreased proenkephalin
A in the cerebrum of CPIP animals seems to reflect the blunted ability to pain modulation and exaggerated response to the pain. For the cereblon, it is known to be related to memory, learning, and intelligence [37], and anomalous cereblon expression can lead to memory and learning deficit [38]. The defect in cereblon gene is associated with mental retardation [39]. Therefore, decreased expression of cereblon in CPIP animals in our study might be related to the deficit in the learning and memory. Neuroserpin, which was known to be related to neurogenesis [40] was decreased in CPIP animals in our study. Neuroserpin plays a role of neuronal protection in pathologic state, and point mutation can cause encephalopathy [41]. Also it has been known that deficiency in neuroserpin exacerbates ischemic brain injury [42]. Therefore, the decreased expression of neuroserpin in CPIP animals in our study might be related to the altered or defected cerebral function.

In our study, we used the CPIP model because CRPS develops after a minor injury without distinguishable nerve lesions. This model is considered a novel animal model of CRPS type 1, in which nerve injury or bone fracture usually does not exist. The previous proteomic studies in neuropathic pain research usually used the nerve ligation model or nerve crush injury model $[43,44]$. Since the CPIP model exhibits similar features of human CRPS type 1, our results may have an implication for cerebral involvement in human CRPS. The mechanical threshold was similar at the beginning (day 1) and after 21 days. This is because we took no actions for treatment on the CPIP animals and therefore the initial pain seemed to persist without change. We did not measure the mechanical threshold in the contralateral paws, because it has 
TABLE 7: Cerebral proteins with decreased expression in the chronic postischemia pain (CPIP) group.

\begin{tabular}{|c|c|c|c|}
\hline Number & Symbol & Description & $P$ value \\
\hline 1 & Vwal & Von Willebrand factor A domain-containing protein 1 & 0.002 \\
\hline 2 & Ppplr10 & Serine/threonine-protein phosphatase 1 regulatory subunit 10 & 0.003 \\
\hline 3 & Poldip2 & DNA-directed polymerase delta interacting protein 2 & 0.003 \\
\hline 4 & Slc4a8 & Isoform 2 of electroneutral sodium bicarbonate exchanger 1 & 0.003 \\
\hline 5 & Daam2 & Dishevelled associated activator of morphogenesis 2 & 0.003 \\
\hline 6 & Cep350 & Centrosome-associated protein 350 & 0.003 \\
\hline 7 & $\operatorname{Tra} 2 \mathrm{~b}$ & Transformer-2 protein homolog beta & 0.007 \\
\hline 8 & Epb4.111 & Isoform $\mathrm{L}$ of band 4.1-like protein 1 & 0.007 \\
\hline 9 & Trim32 & Tripartite motif protein 32 & 0.007 \\
\hline 10 & Slclal & Excitatory amino acid transporter 3 & 0.007 \\
\hline 11 & Spn & Sialophorin & 0.007 \\
\hline 12 & Crbn & Cereblon & 0.007 \\
\hline 13 & Thoc1 & Da2-19 THO complex subunit 1 & 0.007 \\
\hline 14 & $\mathrm{Lmo} 7$ & Lim domain only protein 7 & 0.007 \\
\hline 15 & Rps27a & Ribosomal protein S27a & 0.007 \\
\hline 16 & Sema4d & Sema domain, immunoglobulin domain (Ig), transmembrane domain & 0.043 \\
\hline 17 & Sec3l1 & SEC3-like 1 & 0.047 \\
\hline 18 & Ikbkap & Elongator complex protein 1 & 0.058 \\
\hline 19 & Peci & Peroxisomal 3,2-trans-enoyl-CoA isomerase & 0.058 \\
\hline 20 & Penk & Proenkephalin-A & 0.058 \\
\hline 21 & Bles03 & basophilic leukemia expressed protein & 0.058 \\
\hline 22 & Spna2 & Alpha II spectrin & 0.065 \\
\hline 23 & Pde10a & Isoform 3 of cAMP and cAMP-inhibited cGMP $3^{\prime}, 5^{\prime}$-cyclic phosphodiesterase 10A & 0.065 \\
\hline 24 & Snx2 & Sorting nexin 2 & 0.067 \\
\hline 25 & Slc25a3 & Phosphate carrier protein, mitochondrial & 0.068 \\
\hline 26 & Serpinil & Neuroserpin & 0.070 \\
\hline 27 & Acaalb & Acetyl-CoA acyltransferase $1 \mathrm{~b}$ & 0.077 \\
\hline 28 & $\mathrm{H} 2 \mathrm{afz}$ & Histone H2A.Z & 0.079 \\
\hline 29 & Cox6al & Cytochrome c oxidase subunit $6 \mathrm{~A} 1$, mitochondrial & 0.090 \\
\hline 30 & Acat2 & Acetyl-CoA acetyltransferase 2 & 0.094 \\
\hline
\end{tabular}

been already known that the mechanical threshold decreases in the contralateral paws of the CPIP animals, and ipsilateral plantar allodynia is known to be the most characteristic feature of the CPIP animals [17]. The CMT in the ipsilateral paw was used for the criterion of the successful establishment of the animal model.

This study had some limitations. First, the differentially expressed cerebral proteins may not be specific to CPIP animals. These proteins may also change in response to peripheral noxious stimuli. However, CPIP animals exhibit many features of human CRPS type 1, and thus our findings can be extrapolated to human CRPS. Second, because of the complexity of protein interactions in many physiologic pathways in the brain, it is still unclear which is the key protein in the development of CRPS.

Third, we performed proteomic analysis only 21 days after CPIP model generation. However, protein expression related to the development and maintenance of CRPS can differ according to the time course. A proper time course to evaluate a possible correlation between pain behavior development and protein modulation may be useful to discriminate protein changes associated with the early inflammation from that one responsible for possible structural or functional alterations (neural sensitization) occurring at central level. Further investigation of the cerebral mechanism of CRPS is required.

\section{Conclusion}

In conclusion, the cerebral proteome is altered after CPIP injury; many functional and structural changes seem to occur in the cerebrum. These findings support the notion of cerebral involvement in CRPS. Therefore, treatment of CRPS should target not only the periphery, but also the brain.

\section{Conflict of Interests}

The authors have no conflict of interests to declare.

\section{Acknowledgment}

This study was financially supported by Grant no. 02-2009038 from the Seoul National University Bundang Hospital. 


\section{References}

[1] R. N. Harden, S. Bruehl, M. Stanton-Hicks, and P. R. Wilson, "Proposed new diagnostic criteria for complex regional pain syndrome," Pain Medicine, vol. 8, no. 4, pp. 326-331, 2007.

[2] E. Eisenberg, S. Shtahl, R. Geller et al., "Serum and salivary oxidative analysis in Complex Regional Pain Syndrome," Pain, vol. 138, no. 1, pp. 226-232, 2008.

[3] F. Birklein and M. Schmelz, "Neuropeptides, neurogenic inflammation and complex regional pain syndrome (CRPS)," Neuroscience Letters, vol. 437, no. 3, pp. 199-202, 2008.

[4] J. Schulze and C. Troeger, "Increased sympathetic activity assessed by spectral analysis of heart rate variability in patients with CRPS I," Handchirurgie, Mikrochirurgie, Plastische Chirurgie, vol. 42, pp. 44-48, 2010.

[5] J. Schattschneider, A. Binder, D. Siebrecht, G. Wasner, and R. Baron, "Complex regional pain syndromes: the influence of cutaneous and deep somatic sympathetic innervation on pain," Clinical Journal of Pain, vol. 22, no. 3, pp. 240-244, 2006.

[6] G. L. Moseley, "Distorted body image in complex regional pain syndrome," Neurology, vol. 65, no. 5, p. 773, 2005.

[7] G. L. Moseley, N. Zalucki, F. Birklein, J. Marinus, J. J. van Hilten, and H. Luomajoki, "Thinking about movement hurts: the effect of motor imagery on pain and swelling in people with chronic arm pain," Arthritis Care \& Research, vol. 59, no. 5, pp. 623-631, 2008.

[8] N. E. Acerra and G. L. Moseley, "Dysynchiria: watching the mirror image of the unaffected limb elicits pain on the affected side," Neurology, vol. 65, no. 5, pp. 751-753, 2005.

[9] J. H. Bultitude and R. D. Rafal, "Derangement of body representation in complex regional pain syndrome: report of a case treated with mirror and prisms," Experimental Brain Research, vol. 204, no. 3, pp. 409-418, 2010.

[10] C. S. McCabe, R. C. Haigh, E. F. J. Ring, P. W. Halligan, P. D. Wall, and D. R. Blake, "A controlled pilot study of the utility of mirror visual feedback in the treatment of complex regional pain syndrome (type 1)," Rheumatology, vol. 42, no. 1, pp. 97-101, 2003.

[11] J. Maleki, A. A. LeBel, G. J. Bennett, and R. J. Schwartzman, "Patterns of spread in complex regional pain syndrome, type I (reflex sympathetic dystrophy)," Pain, vol. 88, no. 3, pp. 259266, 2000.

[12] P. Schwenkreis, C. Maier, and M. Tegenthoff, "Functional imaging of central nervous system involvement in complex regional pain syndrome," American Journal of Neuroradiology, vol. 30, no. 7, pp. 1279-1284, 2009.

[13] W. Freund, A. P. Wunderlich, G. Stuber et al., "Different activation of opercular and posterior cingulate cortex (pcc) in patients with complex regional pain syndrome (crps i) compared with healthy controls during perception of electrically induced pain: a functional MRI study," Clinical Journal of Pain, vol. 26, no. 4, pp. 339-347, 2010.

[14] P. Y. Geha, M. N. Baliki, R. N. Harden, W. R. Bauer, T. B. Parrish, and A. V. Apkarian, "The brain in chronic CRPS pain: abnormal gray-white matter interactions in emotional and autonomic regions," Neuron, vol. 60, no. 4, pp. 570-581, 2008.

[15] C. M. Swart, J. F. Stins, and P. J. Beek, "Cortical changes in complex regional pain syndrome (CRPS)," European Journal of Pain, vol. 13, no. 9, pp. 902-907, 2009.

[16] W. Jänig and R. Baron, "Complex regional pain syndrome is a disease of the central nervous system," Clinical Autonomic Research, vol. 12, no. 3, pp. 150-164, 2002.
[17] T. J. Coderre, D. N. Xanthos, L. Francis, and G. J. Bennett, "Chronic post-ischemia pain (CPIP): a novel animal model of complex regional pain syndrome-Type I (CRPS-I; reflex sympathetic dystrophy) produced by prolonged hindpaw ischemia and reperfusion in the rat," Pain, vol. 112, no. 1-2, pp. 94-105, 2004.

[18] S. R. Chaplan, F. W. Bach, J. W. Pogrel, J. M. Chung, and T. L. Yaksh, "Quantitative assessment of tactile allodynia in the rat paw," Journal of Neuroscience Methods, vol. 53, no. 1, pp. 55-63, 1994.

[19] J. K. Eng, A. L. McCormack, and J. R. Yates, "An approach to correlate tandem mass spectral data of peptides with amino acid sequences in a protein database," Journal of the American Society for Mass Spectrometry, vol. 5, no. 11, pp. 976-989, 1994.

[20] W. M. Old, K. Meyer-Arendt, L. Aveline-Wolf et al., "Comparison of label-free methods for quantifying human proteins by shotgun proteomics," Molecular and Cellular Proteomics, vol. 4, no. 10, pp. 1487-1502, 2005.

[21] N. Nadif Kasri, G. Bultynck, I. Sienaert et al., "The role of calmodulin for inositol 1,4,5-trisphosphate receptor function," Biochimica et Biophysica Acta, vol. 1600, no. 1-2, pp. 19-31, 2002.

[22] S. Pezet, F. Marchand, R. D’Mello et al., "Phosphatidylinositol 3-kinase is a key mediator of central sensitization in painful inflammatory conditions," Journal of Neuroscience, vol. 28, no. 16, pp. 4261-4270, 2008.

[23] W.-P. Fung-Leung, "Phosphoinositide 3-kinase delta (PI3K $\delta$ ) in leukocyte signaling and function," Cellular Signalling, vol. 23, no. 4, pp. 603-608, 2011.

[24] P. M. Lorusso and S. A. Boerner, "The role of phosphoinositide 3-kinase in breast cancer: an overview," Clinical Breast Cancer, vol. 10, supplement 3, pp. S56-S58, 2010.

[25] T. Yamauchi, "Neuronal $\mathrm{Ca}^{2+} /$ calmodulin-dependent protein kinase II-discovery, progress in a quarter of a century, and perspective: implication for learning and memory," Biological and Pharmaceutical Bulletin, vol. 28, no. 8, pp. 1342-1354, 2005.

[26] C. Solà, S. Barrón, J. M. Tusell, and J. Serratosa, "The $\mathrm{Ca}^{2+}$ / calmodulin system in neuronal hyperexcitability," The International Journal of Biochemistry \& Cell Biology, vol. 33, no. 5, pp. 439-455, 2001.

[27] A. R. Halt, R. F. Dallapiazza, Y. Zhou et al., "CaMKII binding to GluN2B is critical during memory consolidation," $E M B O$ Journal, vol. 31, no. 5, pp. 1203-1216, 2012.

[28] S. J. Coultrap and K. U. Bayer, "CaMKII regulation in information processing and storage," Trends in Neurosciences, vol. 35, no. 10, pp. 607-618, 2012.

[29] L. Fang, J. Wu, Q. Lin, and W. D. Willis, "Calcium-calmodulindependent protein kinase II contributes to spinal cord central sensitization," The Journal of Neuroscience, vol. 22, no. 10, pp. 4196-4204, 2002.

[30] Y. Dai, H. Wang, A. Ogawa et al., " $\mathrm{Ca}^{2+} /$ calmodulin-dependent protein kinase II in the spinal cord contributes to neuropathic pain in a rat model of mononeuropathy," European Journal of Neuroscience, vol. 21, no. 9, pp. 2467-2474, 2005.

[31] E. D. Crown, Y. S. Gwak, Z. Ye et al., "Calcium/calmodulin dependent kinase II contributes to persistent central neuropathic pain following spinal cord injury," Pain, vol. 153, no. 3, pp. 710-721, 2012.

[32] J. Lisman, R. Yasuda, and S. Raghavachari, "Mechanisms of CaMKII action in long-term potentiation," Nature Reviews Neuroscience, vol. 13, no. 3, pp. 169-182, 2012. 
[33] R. Ruscheweyh, O. Wilder-Smith, R. Drdla, X.-G. Liu, and J. Sandkühler, "Long-term potentiation in spinal nociceptive pathways as a novel target for pain therapy," Molecular Pain, vol. 7, article 20, 2011.

[34] J. Sandkühler and D. Gruber-Schoffnegger, "Hyperalgesia by synaptic long-term potentiation (LTP): an update," Current Opinion in Pharmacology, vol. 12, no. 1, pp. 18-27, 2012.

[35] L. Ferhatovic, A. Banozic, S. Kostic et al., "Expression of calcium/calmodulin-dependent protein kinase II and painrelated behavior in rat models of type 1 and type 2 diabetes," Anesthesia \& Analgesia, vol. 116, no. 3, pp. 712-721, 2013.

[36] F. Luo, C. Yang, Y. Chen et al., "Reversal of chronic inflammatory pain by acute inhibition of $\mathrm{Ca}^{2+} /$ calmodulin-dependent protein kinase II," Journal of Pharmacology and Experimental Therapeutics, vol. 325, no. 1, pp. 267-275, 2008.

[37] J. J. Higgins, A. L. Tal, X. Sun et al., "Temporal and spatial mouse brain expression of cereblon, an ionic channel regulator involved in human intelligence," Journal of Neurogenetics, vol. 24, no. 1, pp. 18-26, 2010.

[38] A. M. Rajadhyaksha, S. Ra, S. Kishinevsky et al., "Behavioral characterization of cereblon forebrain-specific conditional null mice: a model for human non-syndromic intellectual disability," Behavioural Brain Research, vol. 226, no. 2, pp. 428-434, 2012.

[39] J. J. Higgins, J. Pucilowska, R. Q. Lombardi, and J. P. Rooney, "A mutation in a novel ATP-dependent Lon protease gene in a kindred with mild mental retardation," Neurology, vol. 63, no. 10, pp. 1927-1931, 2004.

[40] M. Yaamada, K. Takaehashi, W. Ukai, E. Hashimoto, T. Saito, and M. Yamada, "Neuroserpin is expressed in early stage of neurogenesis in adult rat hippocampus," NeuroReport, vol. 21, no. 2, pp. 138-142, 2010.

[41] E. Miranda and D. A. Lomas, "Neuroserpin: a serpin to think about," Cellular and Molecular Life Sciences, vol. 63, no. 6, pp. 709-722, 2006.

[42] M. Gelderblom, M. Neumann, P. Ludewig et al., "Deficiency in serine protease inhibitor neuroserpin exacerbates ischemic brain injury by increased postischemic inflammation," PLOS ONE, vol. 8, no. 5, Article ID e63118, 2013.

[43] C. R. Jiménez, F. J. Stam, K. W. Li et al., "Proteomics of the injured rat sciatic nerve reveals protein expression dynamics during regeneration," Molecular and Cellular Proteomics, vol. 4, no. 2, pp. 120-132, 2005.

[44] O. Alzate, S.-R. A. Hussain, V. M. Goettl et al., "Proteomic identification of brainstem cytosolic proteins in a neuropathic pain model," Molecular Brain Research, vol. 128, no. 2, pp. 193200, 2004. 

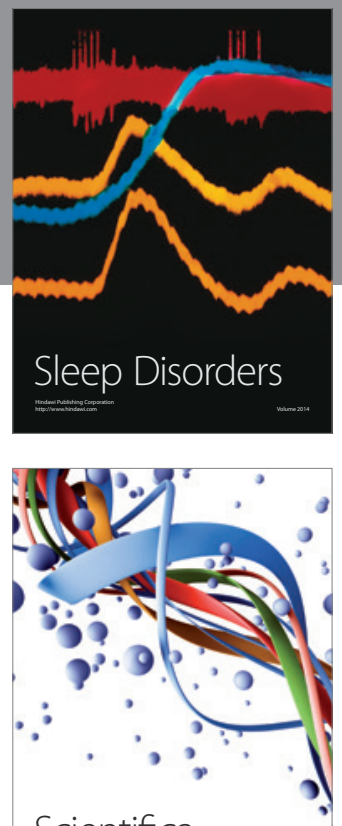

Scientifica
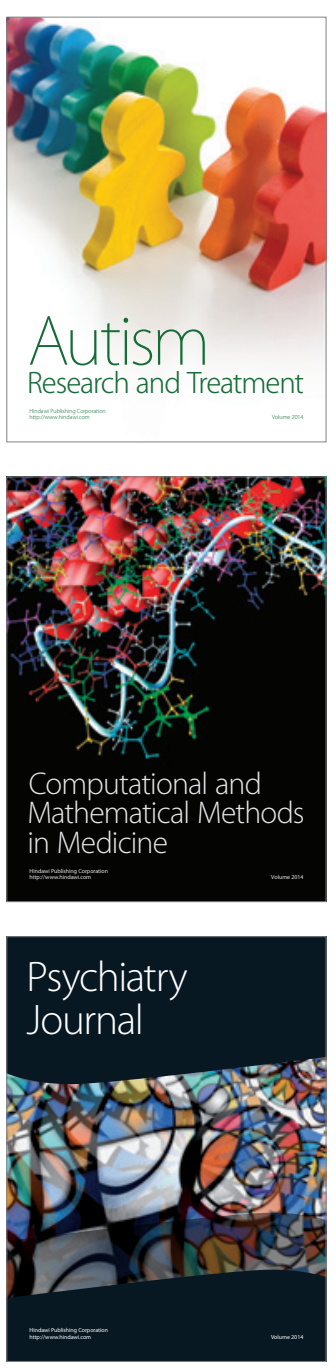
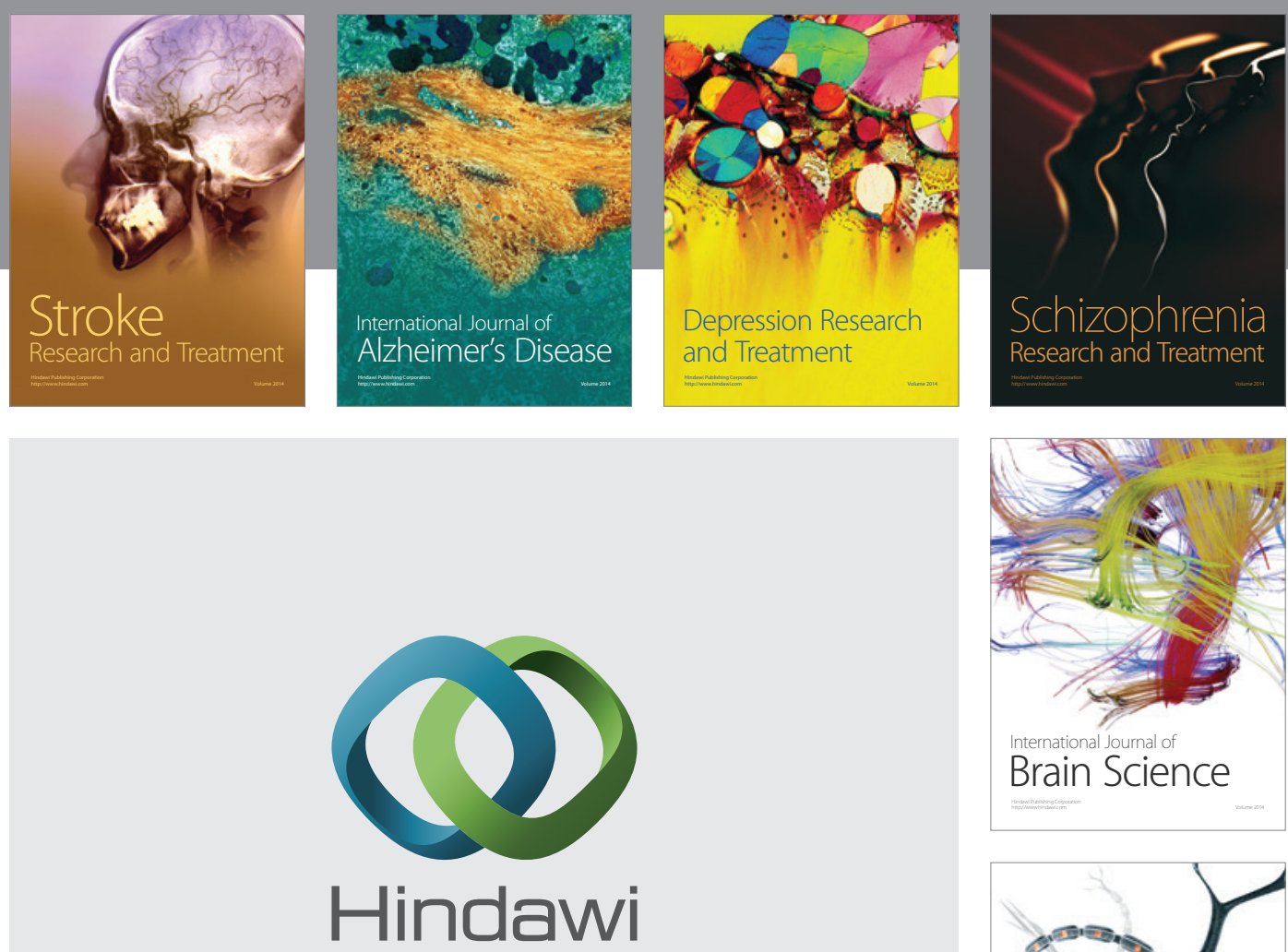

Submit your manuscripts at

http://www.hindawi.com
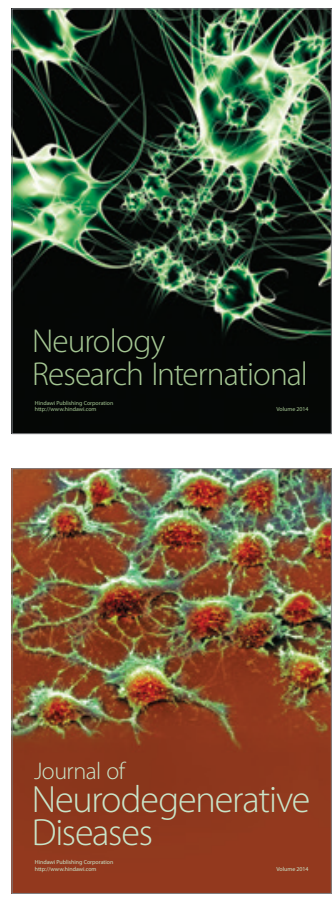

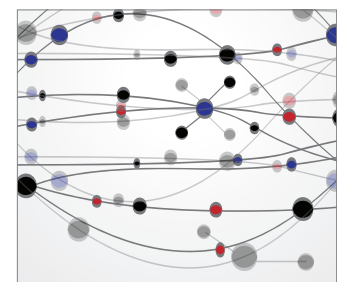

The Scientific World Journal
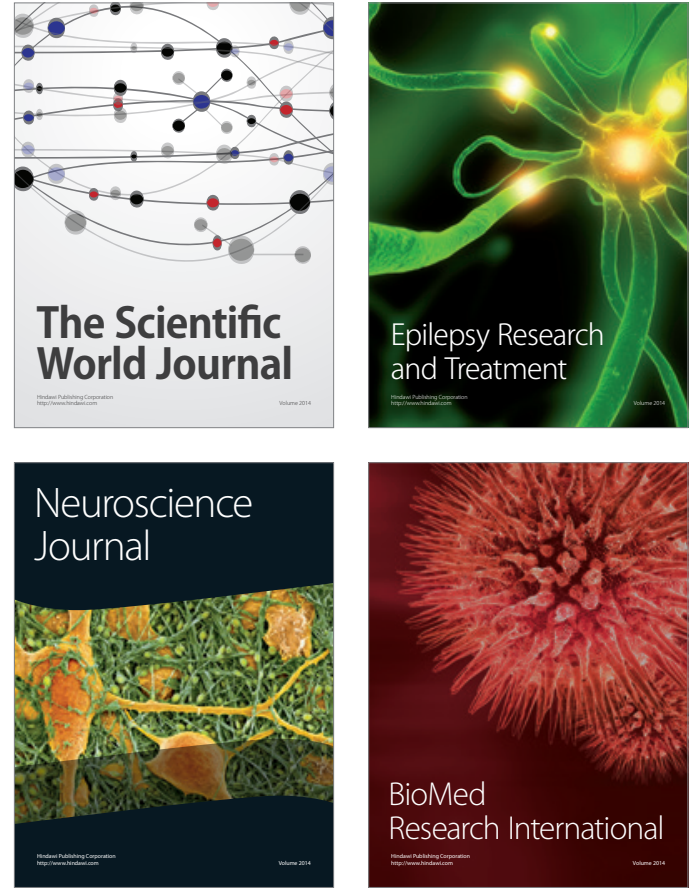

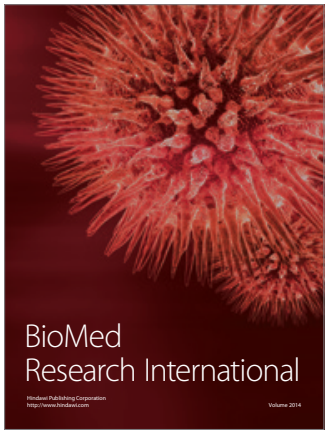

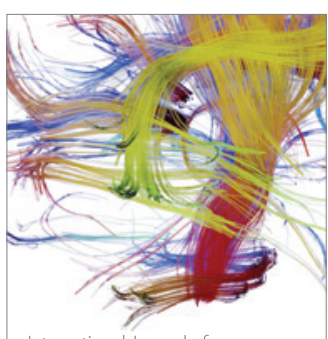

Brain Science

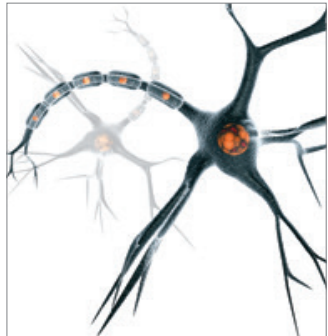

Neural Plasticity
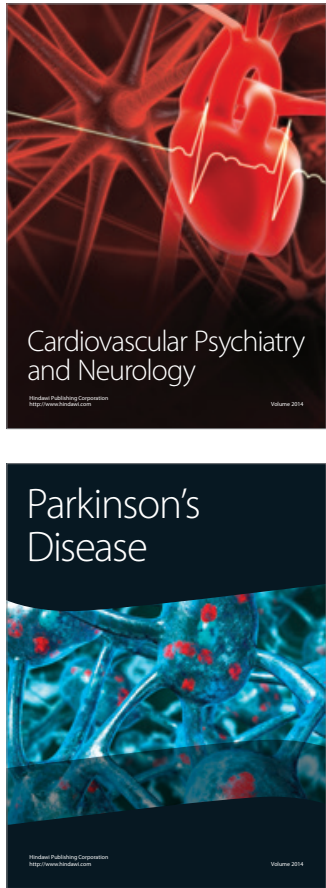